\title{
Detection of staphylococcal antibodies by gel diffusion
}

\author{
D. M. STANDRING \\ From the Department of Pathology, Institute of Orthopaedics, \\ Royal National Orthopaedic Hospital, Stanmore, Middlesex
}

sYNOPSIS A simple method using the Elek-Ouchterlony gel diffusion method is described for the detection of anti-alpha haemolysin and anti-leucocidin antibodies. Two hundred and forty-six sera from patients known or suspected to have staphylococcal infection and 48 normal sera from antenatal cases are included in the survey. It is evident that the gel diffusion method compares favourably with the haemagglutination method for detecting anti-leucocidin. The conventional tube method still has advantages of speed and accuracy in the estimation of anti-alpha haemolysin, as the diagnostic reagent used contains other antigens which confuse the picture in gel diffusion.

Since the publication of papers on the measurement of staphylococcal antibodies from this department (Lack, 1957; Towers and Gladstone, 1958; Towers, 1961 ; Lack and Towers, 1962), we have been doing tests for hospitals throughout the country. Thus it has become apparent that the need for serological tests for the diagnosis of staphylococcal infection is widespread. In order to simplify the tests and economize on the antigens, the Elek-Ouchterlony gel diffusion technique was tried, and a comparison is now made between the results obtained using this technique and the tube tests for measuring the antibodies to alpha haemolysin and leucocidin.

\section{MATERIALS AND METHODS}

ALPHA HAEMOLYSIN Burroughs Wellcome diagnostic reagent was used, diluted with $0.2 \%$ gelatin saline to a strength of 40 units $/ \mathrm{ml}$.

LEUCOCIDIN Crude leucocidin slurry was obtained from the Microbiological Research Establishment, Porton, Wilts., and further purified by Woodin's method(Woodin, 1961). The fraction used for the gel diffusion technique contained the $F$ component of leucocidin contaminated with a little of the $S$ component and was diluted 1 in $\mathbf{4 0}$ with physiological saline.

AGAR Oxoid ionagar no. 2, $1.2 \%$, sodium azide, $1 \%$ and $30 \mathrm{mg}$. E.D.T.A. in $100 \mathrm{ml}$. distilled water were dissolved by heat and sterilized at $10 \mathrm{lb}$./sq. in. for 10 minutes.

Received for publication 10 January 1964
SERA Two hundred and forty-six sera were used from patients suspected or known to have staphylococcal infection, in the majority of cases the diagnosis being osteomyelitis. These sera had been kept at $-15^{\circ} \mathrm{C}$. for varying periods of time from 12 months to four years. Forty-eight ante-natal sera were included in the survey as a normal series; the past histories of these patients are not known. All the sera were inactivated at $56^{\circ} \mathrm{C}$. for 30 minutes.

PREPARATION OF AGAR SLIDES One drop of the molten agar was spread in a thin film over the surface of a clean dry 3 in. $\times 1$ in. glass slide and allowed to dry in air, and then a further $2.5 \mathrm{ml}$. of agar was added to the slide and allowed to set. Wells in the agar (Fig. 1) were made using an agar cutter. The cutter was pressed into the gel, lifted away, and the resultant agar plugs removed using a capillary tube attached to a water vacuum pump.

The peripheral wells were filled with patient's serum and the central well with antigen using a fine bore

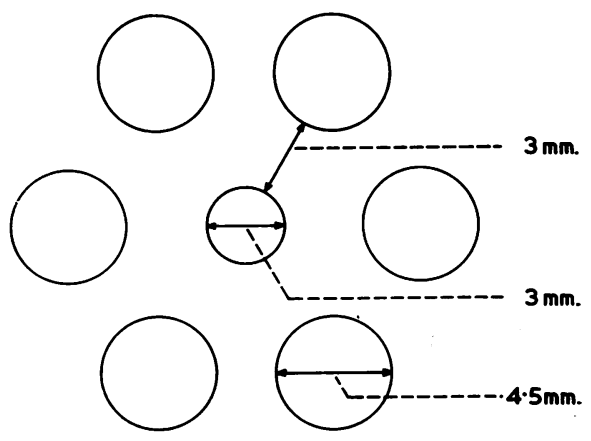


Pasteur pipette and the slides then housed in a wet chamber, kept at room temperature and examined after 24 and 48 hours. No change was noted between the 24-hour reading and that at 48 hours.

TUBE TESTS The anti-alpha haemolysin test and the haemagglutination tests for anti-leucocidin were carried out as described by Lack and Towers (1962). The upper limit of normal for the anti-alpha haemolysin test is 2 units/ml., and that for the anti-leucocidin test is 8 . (As very high dilutions of patients' serum are used for this test, this figure is used for the sake of convenience, in preference to $1 / 8,000$.)

\section{RESULTS}

In reading the gel diffusion results, the terms 'weak line', 'definite line', and 'strong line' are used to denote the increasing intensities of the precipitation lines. In some cases more than one line was produced by the antigen-antibody reaction, but as the antigens used are known to contain other staphylococcal toxins, these lines were all taken as evidence of staphylococcal infection, and the strongest reaction was chosen to be representative of the results (Tables I and II).

TABLE I

\begin{tabular}{lcr}
\multicolumn{3}{c}{ ANTI-ALPHA HAEMOLYSIN } \\
Gel Diffusion Results & $\begin{array}{l}\text { Tube Results } \\
\text { (units/ml. })\end{array}$ \\
\hline & $<2$ & $34(63 \cdot 0 \%)$ \\
No precipitation line & $2-4$ & $5(9 \cdot 3 \%)$ \\
(54 cases) & $4-8$ & $8(14.8 \%)$ \\
& $>8$ & $7(12.9 \%)$ \\
Weak line & $<2$ & $36(55 \cdot 4 \%)$ \\
(65 cases) & $2-4$ & $13(20 \cdot 0 \%)$ \\
& $4-8$ & $12(18 \cdot 4 \%)$ \\
Definite line & $>8$ & $4(6 \cdot 2 \%)$ \\
(117 cases) & $<2$ & $22(18 \cdot 8 \%)$ \\
& $2-4$ & $21(18 \cdot 0 \%)$ \\
Strong line & $4-8$ & $18(15 \cdot 4 \%)$ \\
(10 cases) & $>8$ & $56(47 \cdot 8 \%)$ \\
& $<2$ & - \\
& $2-4$ & - \\
& $4-8$ & $10(100 \%)$
\end{tabular}

Total number of cases $=246$

1The number of sera with a titre of less than 2 units $/ \mathrm{ml}$. by the tube method which gave a line using the gel diffusion method was 58 or $24 \%$ of the total; those with titres of over 2 units $/ \mathrm{ml}$. (i.e. raised values) which failed to give a line using the gel diffusion method was 20 or $8 \%$ of the total.

Fifty of the sera known to produce precipitation lines were then diluted (from neat to 1/16) and the gel diffusion repeated to see if any correlation could be found between the tube titres and the gel diffusion titres.

The end-point was taken as the highest dilution which formed a precipitation line with the antigen.
TABLE II

ANTI-LEUCOCIDIN ${ }^{1}$

\begin{tabular}{lcr} 
Gel Diffusion Results & $\begin{array}{c}\text { Tube Results } \\
\text { (serum titre) }\end{array}$ & \\
\hline & $<4$ & $5(19 \cdot 2 \%)$ \\
No precipitation line & $4-8$ & $14(53 \cdot 9 \%)$ \\
(26 cases) & $16-32$ & $6(23 \cdot 1 \%)$ \\
& 64 and over & $1(3 \cdot 8 \%)$ \\
Weak line & 4 & $1(6 \cdot 25 \%)$ \\
(16 cases) & $4-8$ & $4(25 \%)$ \\
& $16-32$ & $10(62 \cdot 5 \%)$ \\
Definite line & 64 and over & $1(6 \cdot 25 \%)$ \\
(77 cases) & $<4$ & $1(1 \cdot 3 \%)$ \\
& $4-8$ & $19(24 \cdot 7 \%)$ \\
Strong line & $16-32$ & $31(40 \cdot 2 \%)$ \\
(127 cases) & 64 and over & $26(33 \cdot 8 \%)$ \\
& $<4$ & $2(1 \cdot 6 \%)$ \\
& $4-8$ & $15(11 \cdot 8 \%)$ \\
& $16-32$ & $49(38 \cdot 6 \%)$
\end{tabular}

Total number of cases $=246$

${ }^{1}$ The number of sera with titres of 8 or under which gave a line using $\int$ the gel diffusion method was 42 or $17 \%$ of the total; those with titres of over 8 (i.e., raised values) which failed to give a line using the gel $\stackrel{\square}{\square}$ diffusion method was seven or $3 \%$ of the total.

TABLE III

ANTI-ALPHA HAEMOLYSIN

Gel Diffusion Tube Tests (units/ml.)

\section{Line only with neat serum} (8 cases)

2
$2-4$

4-8

$>8$

$<2$

Line at $1 / 2$ dilution

(8 cases)

$2-4$

4-8

$>8$

$<2$

Line at $1 / 4$ dilution

(13 cases)

$2-4$

4-8

$>8$

$<2$

Line at $1 / 8$ dilution

(11 cases)

4-8

$>8$

$<2$

Line at $1 / 16$ dilution

(10 cases)

2-4

$>8$

Total number of cases $=50$

\begin{tabular}{l}
4 \\
2 \\
2 \\
\hline \\
\hline 4 \\
2 \\
2 \\
3 \\
2 \\
1 \\
7 \\
4 \\
2 \\
3 \\
2 \\
3 \\
2 \\
1 \\
4
\end{tabular}

Again it was found that more than one line was formed by some of the sera (Tables III and IV).

ANTI-ALPHA HAEMOLYSIN All the 48 control sera from ante-natal patients proved to have titres of less than 2 units $/ \mathrm{ml}$. by the tube method. Two sera produced $\stackrel{\Phi}{\Phi}$ weak lines by the gel diffusion method and two other sera produced definite lines. The remaining 44 sera failed to produce precipitation lines. The two sera $\frac{\mathbb{D}}{\mathrm{D}}$ producing definite lines gave lines at $1 / 4$ dilution. They were negative at $1 / 8$ dilution. 
TABLE IV

ANTI-LEUCOCIDIN

Gel Diffusion

Tube Tests (serum titre)

Line only with neat serum

( 9 cases)

Line at $1 / 2$ dilution

(10 cases)

Line at $1 / 4$ dilution

(12 cases)

Line at $1 / 8$ dilution (10 cases)

Line at $1 / 16$ dilution ( 9 cases)

$\begin{array}{ll}<4 & - \\ 4-8 & 7 \\ 16-32 & 2 \\ 64 \text { and over } & - \\ <4 & - \\ 4-8 & 3 \\ 16-32 & 6 \\ 64 \text { and over } & 1 \\ <4 & 1 \\ 4-8 & 3 \\ 16-32 & 4 \\ 64 \text { and over } & 4 \\ <4 & - \\ 4-8 & - \\ 16-32 & 4 \\ 64 \text { and over } & 6 \\ 4 & - \\ 4-8 & - \\ 16-32 & - \\ 64 \text { and over } & 9\end{array}$

Total number of cases $=\mathbf{5 0}$

ANTI-LEUCOCIDIN All the 48 control sera gave titres of 4 or less by the haemagglutination method. One serum gave a weak line by the gel diffusion method and six other sera produced definite lines. The remaining 41 sera failed to produce precipitation lines. Of the six sera which produced definite lines, two gave a line at neat only and four gave a line at $1 / 2$ dilution. All were negative at $1 / 4$ dilution.

\section{CONCLUSIONS}

The gel diffusion method is time saving in prepar- ation compared with the tube methods, but unlike the conventional anti-alpha haemolysin titration, the results are not known until the following day. Sera diluted above $1 / 4$ giving a line against leucocidin correlate well with a raised anti-leucocidin titre by haemagglutination. In the case of anti-alpha haemolysin titration, the gel diffusion results did not correlate so well with the tube results. Of the 50 sera which were titrated, 10 with a titre of less than 2 units $/ \mathrm{ml}$. (by the tube method) of anti-alpha haemolysin gave a line at $1 / 4$ dilution or higher, three of them going as high as $1 / 16$. However, it was noted that eight of these 10 sera gave a raised antileucocidin titre by haemagglutination, and, as the toxin contains other staphylococcal toxins, the lines produced may be leucocidin-anti-leucocidin reactions.

Using gel diffusion methods the anti-leucocidin appears more helpful than the anti-alpha haemolysin, especially when taken to titre.

My thanks are due to Dr. C. H. Lack for his supervision and advice, and to the Microbiological Research Establishment, Porton, for supplying crude leucocidin slurry.

This work was made possible by a grant from the Clinical Research Fund, Royal National Orthopaedic Hospital.

REFERENCES

Lack, C. H. (1957). Proc. roy. Soc. Med., 50, 625.

-, and Towers, A. G. (1962). Brit. med. J., 2, 1227. Towers, A. G. (1961). J. clin. Path., 14, 161.

-, and Gladstone, G. P. (1958). Lancet, 2, 1192.

Woodin, A. M. (1961). J. Path. Bact., 81, 63. 